\title{
Optical anisotropy in type-II ZnTe/ZnSe submonolayer quantum dots
}

H. Ji, S. Dhomkar, R. Wu, V. Shuvayev, V. Deligiannakis, M. C. Tamargo, J. Ludwig, Z. Lu, D. Smirnov, A. Wang, and I. L. Kuskovsky

Citation: Journal of Applied Physics 119, 224306 (2016); doi: 10.1063/1.4953675

View online: https://doi.org/10.1063/1.4953675

View Table of Contents: http://aip.scitation.org/toc/jap/119/22

Published by the American Institute of Physics

\section{Articles you may be interested in}

Spin dynamics of ZnSe-ZnTe nanostructures grown by migration enhanced molecular beam epitaxy Journal of Applied Physics 121, 115702 (2017); 10.1063/1.4978314

Properties of photoluminescence in type-II ZnTe / ZnSe quantum dots Applied Physics Letters 88, 121917 (2006); 10.1063/1.2189029

High color purity ZnSe/ZnS core/shell quantum dot based blue light emitting diodes with an inverted device structure

Applied Physics Letters 103, 053106 (2013); 10.1063/1.4817086

Determination of shape anisotropy in embedded low contrast submonolayer quantum dot structures

Applied Physics Letters 107, 251905 (2015); 10.1063/1.4938399

Band parameters for III-V compound semiconductors and their alloys

Journal of Applied Physics 89, 5815 (2001); 10.1063/1.1368156

Design of cadmium-free colloidal II-VI semiconductor quantum dots exhibiting RGB emission

AIP Advances 7, 045309 (2017); 10.1063/1.4982256

\section{AIP |roumal af}

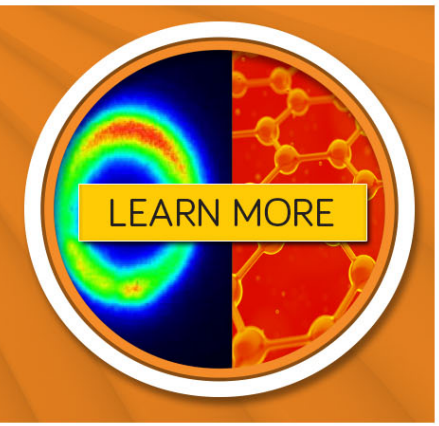




\title{
Optical anisotropy in type-II ZnTe/ZnSe submonolayer quantum dots
}

\author{
H. Ji, ${ }^{1,3}$ S. Dhomkar, ${ }^{1,3}$ R. Wu, ${ }^{1,3}$ V. Shuvayev, ${ }^{1}$ V. Deligiannakis, ${ }^{2,3}$ M. C. Tamargo, ${ }^{2,3}$ \\ J. Ludwig, ${ }^{4,5}$ Z. Lu, ${ }^{4,5}$ D. Smirnov, ${ }^{4}$ A. Wang, ${ }^{6}$ and I. L. Kuskovsky ${ }^{1,3}$ \\ ${ }^{1}$ Department of Physics, Queens College of CUNY, Queens, New York 11367, USA \\ ${ }^{2}$ Department of Chemistry, City College of CUNY, New York, New York 10031, USA \\ ${ }^{3}$ The Graduate Center of CUNY, New York, New York 10016, USA \\ ${ }^{4}$ National High Magnetic Field Laboratory, Tallahassee, Florida 32310, USA \\ ${ }^{5}$ Department of Physics, Florida State University, Tallahassee, Florida 32306, USA \\ ${ }^{6}$ Evans Analytical Group, Sunnyvale, California 94086, USA
}

(Received 24 January 2016; accepted 30 May 2016; published online 14 June 2016)

Linearly polarized photoluminescence is observed for type-II ZnTe/ZnSe submonolayer quantum dots (QDs). The comparison of spectral dependence of the degree of linear polarization $(D L P)$ among four samples indicates that the optical anisotropy is mostly related to the elongation of ZnTe QDs. Numerical calculations based on the occupation probabilities of holes in $p_{x}$ and $p_{y}$ orbitals are performed to estimate the lateral aspect ratio of the QDs, and it is shown that it varies between 1.1 and 1.4. The value of anisotropic exchange splitting for bright excitonic states is found to be $\sim 200 \mu \mathrm{eV}$ from the measurement of the degree of circular polarization as a function of the magnetic field. The results also show that heavy-light hole mixing ratio is about 0.16. Published by AIP Publishing. [http://dx.doi.org/10.1063/1.4953675]

\section{INTRODUCTION}

Semiconductor quantum dots (QDs) are threedimensional nanostructures in which both carriers (type-I) or one of them (type-II) is confined along the three dimensions of space by the band gap difference between the dot and barrier materials. QDs have been proposed as candidates for a number of applications, including information processing, ${ }^{1-3}$ and especially for quantum computation with optical control. $^{4,5}$ The characterization, understanding, and control of the shape or strain anisotropy of QDs are important since they strongly influence the optical properties of the devices. ${ }^{6,7}$ Generally, a lowering of confinement symmetry in QDs will lead to the valence band mixing between heavy hole and light hole states,${ }^{8-11}$ which are originally separated due to confinement. Consequently, the two degenerate circularly polarized bright exciton states $|+1\rangle$ and $|-1\rangle$ will mix and form nondegenerate linearly polarized states $\frac{1}{\sqrt{2}}(|+1\rangle \pm|-1\rangle)$, the emissions from which are polarized along the [110] and [1 10$]$ crystal directions, respectively, ${ }^{12,13}$ forming a so-called doublet fine structure. ${ }^{13,14}$ Such linearly polarized emissions have been observed and studied in InAs/GaAs, ${ }^{713-15}$ InGaAs/ GaAs, ${ }^{16,17} \mathrm{InAs} / \mathrm{InP},{ }^{11} \mathrm{InP} / \mathrm{InGaP},{ }^{18,19} \mathrm{CdTe} / \mathrm{ZnTe},{ }^{20}$ and $\mathrm{CdSe} / \mathrm{ZnSe}^{21}$ Stranski-Krastanov (SK) QDs, but rarely reported for submonolayer QDs. ${ }^{22}$ Polarizations along $[110]^{19,21}$ and $[1 \overline{1} 0]^{18,23}$ directions are both reported. ${ }^{24}$ Three main origins of confinement symmetry lowering have been proposed: ${ }^{13}$ (i) structural elongation of the QDs, ${ }^{14,16,19,23-27}$ (ii) anisotropic strain relief or defects, ${ }^{13,23,28}$ and (iii) anisotropic interface bond alignment. ${ }^{29}$

We focus on ZnSe-based layers with ZnTe-rich submonolayer quantum dot multilayers for the capability of tuning type-II band alignment, ${ }^{30-32}$ and controlling the QD size and density, which is a desirable advantage in optical applications. $^{23,34}$ The submonolayer nature of the QDs is achieved through migration enhanced epitaxy (MEE), presenting remarkable features including the absence of wetting layers and significantly smaller dimensions compared to SK QDs. ${ }^{33,34}$ Although very recently ${ }^{35}$ elongation of $\mathrm{ZnTe} / \mathrm{ZnSe}$ QDs has been reported as observed in high resolution $\mathrm{x}$-ray diffraction (HRXRD) experiments, detailed investigation of the possible mechanisms leading to optical anisotropy has not been yet discussed for this system.

Herein, we report the optical anisotropy in type-II ZnTe/ ZnSe submonolayer QDs observed via both linear polarization of photoluminescence (PL) and circular polarization of magneto-PL. Through analysis of the degree of linear polarization $(D L P)$, we calculated the ratio of heavy-light hole mixing. Combined with the magnetic field dependence of the degree of circular polarization $(D C P)$, we obtained the energy of anisotropic exchange splitting for type-II excitons in this system. Based on the spectral dependence of $D L P$ for the four samples with varied average Te concentrations and different strains, we propose that the optical anisotropy is mostly related to structure elongation of the ZnTe QDs. Furthermore, we performed numerical calculations, based on the occupation probabilities of holes in $p_{x}$ and $p_{y}$ orbitals, which we compare with the experimentally observed $D L P S$ in order to estimate the aspect ratio of the elongated QDs.

\section{EXPERIMENTAL RESULTS AND DISCUSSION}

The ZnSe layers with ZnTe sub-monolayer QDs were grown in a Riber 2300P molecular beam epitaxy (MBE) system on (001) GaAs substrates by a combination of MEE and $\mathrm{MBE}$ as reported in Ref. 36 and references therein. By varying the Te cell temperature, four samples were grown using different Te fluxes during the formation of QDs. All other growth conditions remained the same. The samples are listed in Table I, along with the growth parameters, the average compressive strain, and the Te content obtained via secondary ion mass spectrometry (SIMS) performed by Evans 
TABLE I. Growth parameters, strain, Te content, QD size, and density in the samples. ${ }^{34}$

\begin{tabular}{lcccc}
\hline \hline Samples & A & B & C & D \\
\hline Number of periods & 100 & 250 & 100 & 120 \\
Super lattice period in nm & 1.7 & 2.5 & 1.7 & 2.8 \\
Compressive strain in ppm & $3 \times 10^{3}$ & $1 \times 10^{3}$ & $6 \times 10^{3}$ & $3 \times 10^{3}$ \\
Total Te content from SIMS in \% & 0.1 & 0.3 & 0.5 & 3.2 \\
Average QD radius in nm & 13.4 & 15.1 & 15.3 & 19.7 \\
Average QD thickness in nm & 0.40 & 0.45 & 0.51 & 1.00 \\
Average QD areal density in $10^{9} \mathrm{~cm}^{-2}$ & 1.6 & 3.8 & 3.5 & 12.4 \\
\hline \hline
\end{tabular}

Analytical Group. Samples A, B, C, and D are organized in order of increasing Te content. The QD sizes and densities reported in Ref. 34 are also listed.

For back-scattering linearly polarized PL measurements, a $405 \mathrm{~nm}$ diode laser, combined with a linear polarizer and a quarter-wave plate, was used to excite the samples with the right-handed circularly polarized light. The emission from the samples was focused onto a fiber coupled to an OceanOptics high resolution solid state spectrometer. Samples were kept in an ARS, Inc., temperature-variable closed-cycle refrigerating system, allowing for measurements at $7.5 \mathrm{~K}$. A linear polarizer on a rotating mount was placed in front of the collecting fiber to analyze the linear polarization of the PL. Circularly polarized magneto-PL measurements were performed in the Faraday geometry within an $18 \mathrm{~T}$ superconducting magnet in the National High Magnetic Field Laboratory (NHMFL). Excitation was achieved by injecting emission from a $405 \mathrm{~nm}$ Thorlabs temperature controlled laser diode into a $365 \mu \mathrm{m}$ optical fiber and delivered to the sample in a ${ }^{3} \mathrm{He}$ cryostat. Optical power density across the excitation spot was fixed to $\sim 10 \mu \mathrm{W} / \mathrm{cm}^{2}$. The PL was analyzed by a circular polarizer consisting of an achromatic quarter wave plate and a linear polarizer. The $\sigma^{+}$ and $\sigma^{-}$circularly polarized PL components were selected by reversing the polarity of the magnetic field. The PL was collected via a $550 \mu \mathrm{m}$ fiber and delivered to a Princeton Instruments IsoPlane single grating spectrometer equipped with a thermoelectrically cooled CCD detector.

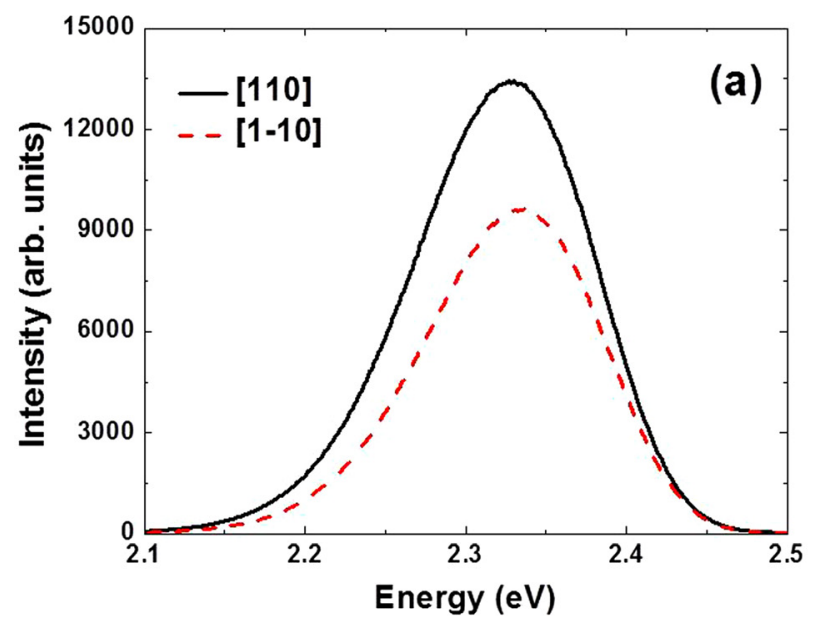

The PL spectra of sample D polarized along the [110] and [1 10$]$ axes are shown in Figure 1(a), while the spectrally integrated PL intensity as a function of the angle between axis of the linear polarizer and the [110] axis is shown in Figure 1(b). The [110] polarized emission is the strongest, whereas the $[1 \overline{1} 0]$ polarized one is the weakest. This agrees with the structural elongation of QDs along [110] axis obtained from HRXRD experiments. ${ }^{35}$ The $D L P$, defined as $\frac{I_{[110]}-I_{[1-10]}}{I_{[110]}+I_{[1-10]}}$, is $\sim 0.18$.

Since the PL spectrum is broad and consists of multiple bands, ${ }^{36}$ the doublet fine structure cannot be resolved from the linearly polarized spectra. Thus, to obtain the value of the anisotropic exchange splitting, we studied the $D C P$ of the PL as a function of the magnetic field for sample D. Without the magnetic field, as discussed above, the PL emission is linearly polarized due to the anisotropic exchange splitting. With an increasing magnetic field, the Zeeman splitting gradually increases and eventually dominates the anisotropic exchange splitting, resulting in decreased mixing between the $|+1\rangle$ and $|-1\rangle$ excitonic states. Therefore, the $D C P$ will increase with the increasing magnetic field. The $D C P$ as a function of the square of magnetic field is shown in Figure 2.

To analyze these results, we follow Refs. 12 and 37, where the magnetic field dependence of the $D C P$ is discussed in terms of competition among the Zeeman splitting, the anisotropic exchange splitting, and the bright/dark exciton relaxation; such dependence can be described by the following expression: ${ }^{37}$

$$
D C P(B)=\frac{B^{2}}{B^{2}+B_{1}^{2}}\left[P_{c}^{0}+\tilde{P}_{c}^{0} \frac{B^{2}}{B^{2}+B_{2}^{2}}\right],
$$

where the effective magnetic fields $B_{1}=\frac{\Delta E_{1}}{\Delta E_{Z B} / B}$ and $B_{2}$ $=\frac{d^{1 / 2} \Delta E_{2}}{\Delta E_{Z D} / B}$ stand for the ratio between anisotropic exchange splitting $\left(\Delta E_{1}\right.$ and $\left.\Delta E_{2}\right)$ and Zeeman splitting $\left(\Delta E_{Z B}\right.$ and $\left.\Delta E_{Z D}\right)$ of the bright and the dark excitonic states, respectively, $d>1$ is a constant, and $P_{c}^{0}$ and $\tilde{P}_{c}^{0}$ are constants. Constant $P_{c}^{0}$ can be related to the ratio of light-heavy hole mixing, $\tilde{\gamma}$, via the expression $P_{c}^{0}=\left(1-\tilde{\gamma}^{2} / 3\right) /\left(1+\tilde{\gamma}^{2} / 3\right)$ introduced in Ref. 9 .

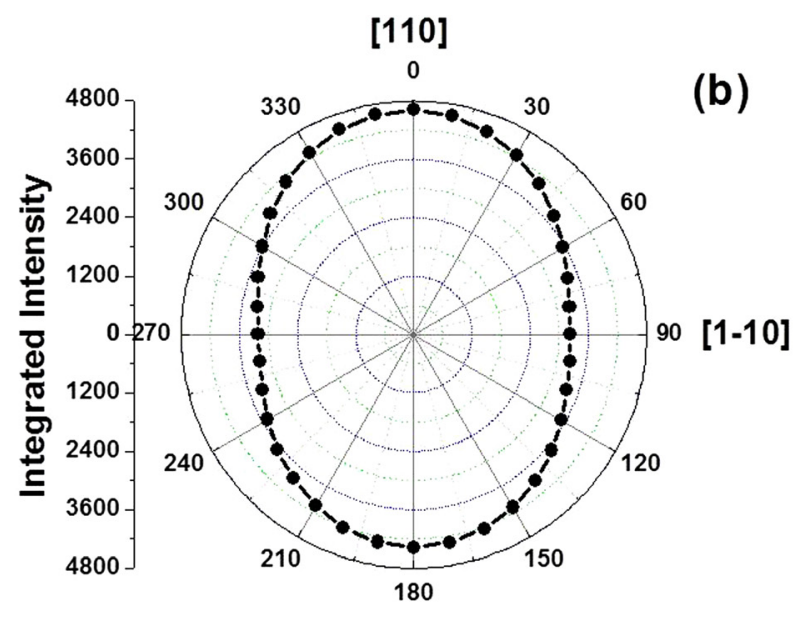

FIG. 1. (a) The photoluminescence emission of sample D polarized along [110] (solid line) and [1 $1 \overline{1} 0]$ (dashed line) crystal axes. (b) The integrated intensity of the photoluminescence of sample D as a function of the angle between axis of the linear polarizer and the [110] crystal axis. 


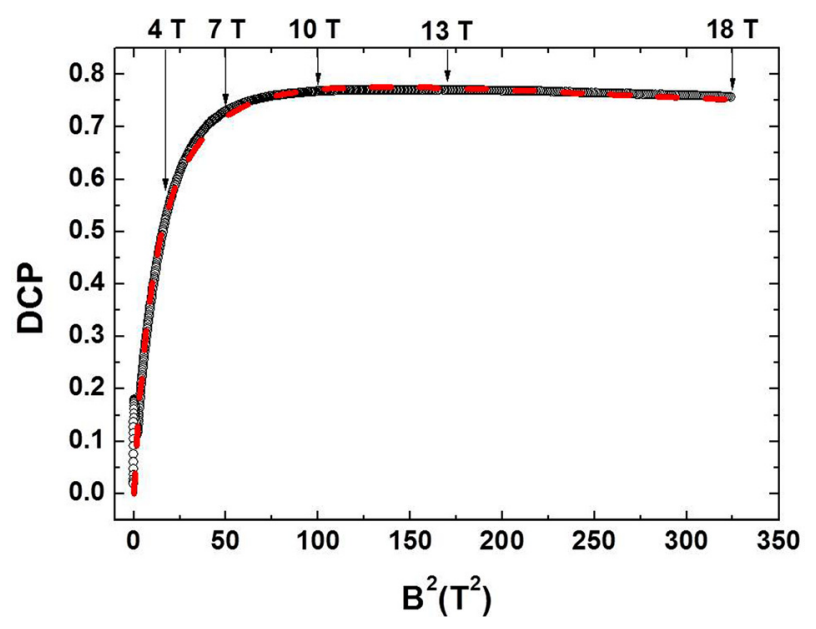

FIG. 2. Degree of circular polarization (open circles) as a function of the square of magnetic field for sample D. The dashed line is fitted to Eq. (1).

At the same time, values of $\tilde{\gamma}$ can be deduced from the $D L P$ according to the following relation: 8,9

$$
D L P=2 \kappa \tilde{\gamma} /\left(1+\kappa^{2} \tilde{\gamma}^{2}\right),
$$

where $\kappa=1 / \sqrt{3}$ measures the difference in strength between light hole and heavy hole radiative coupling. Thus, from the $D L P$ we calculated the overall ratio of light-heavy hole mixing in sample $\mathrm{D}$, which is $\sim 0.16$. Therefore, $P_{c}^{0}$ is $\sim 0.98$ for sample $\mathrm{D}$. It can be shown ${ }^{38}$ that $\Delta E_{Z B}$ and $\Delta E_{Z D}$ in our system are determined only by the electron Zeeman splitting, and therefore are given by $g_{e} \mu_{B} B$ (here $g_{e}$ is the electron $g$-factor and $\mu_{B}$ is the Bohr magneton). Fitting the data (open circles) in Figure 2 to Eq. (1) gives $\tilde{P}_{c}^{0}=-0.34$, $B_{1}=3.8 \mathrm{~T}$ and $B_{2}=14 \mathrm{~T}$, which translates to the anisotropic exchange splitting of $\Delta E_{1}=0.22 \mathrm{meV}$ for bright excitons, and $\Delta E_{2}=0.81 \mathrm{meV}$ for dark excitons. These values of anisotropic exchange splitting are comparable to those reported for CdTe and CdSe QDs (0-0.5 meV, see Refs. 20, 21 and references therein). We note that at very low fields (Figure 2) there are vertical series of data points, which do not match the fitting curve. These are related to the "initial drop" and the excitonic Aharonov-Bohm peak that were previously reported for these type-II ZnTe/ZnSe submonolayer QDs (see, e.g., Refs. 29 and 36), and which do not affect the overall fitting.

Next, we discuss the origin of the observed optical anisotropy. Ivchenko and Nestoklen ${ }^{29}$ have suggested that the optical anisotropy of type-II heterostructures $\mathrm{CA} / \mathrm{C}^{\prime} \mathrm{A}^{\prime}$ can occur from anisotropic interface bond alignment since the relative contributions of the $p_{x^{-}}$and $p_{y}$-orbitals to the valence-band function near the interface $\mathrm{C}-\mathrm{A}^{\prime}$ or $\mathrm{C}^{\prime}-\mathrm{A}$ differ substantially. However, for the ZnTe/ZnSe QD system, the interface can only be Te-Zn-Se. Therefore, the interfacial symmetry lowering is not the cause of the optical anisotropy in our samples. To distinguish between the contribution from the anisotropic strain relief or defects and the QDs structure elongation, we investigated the spectral dependence of the $D L P$ for the four samples, as plotted in Figure 3, overlaid with their normalized PL spectra at $7.5 \mathrm{~K}$. Detailed optical analysis of the samples (see Refs. 32, 36, and 39 and

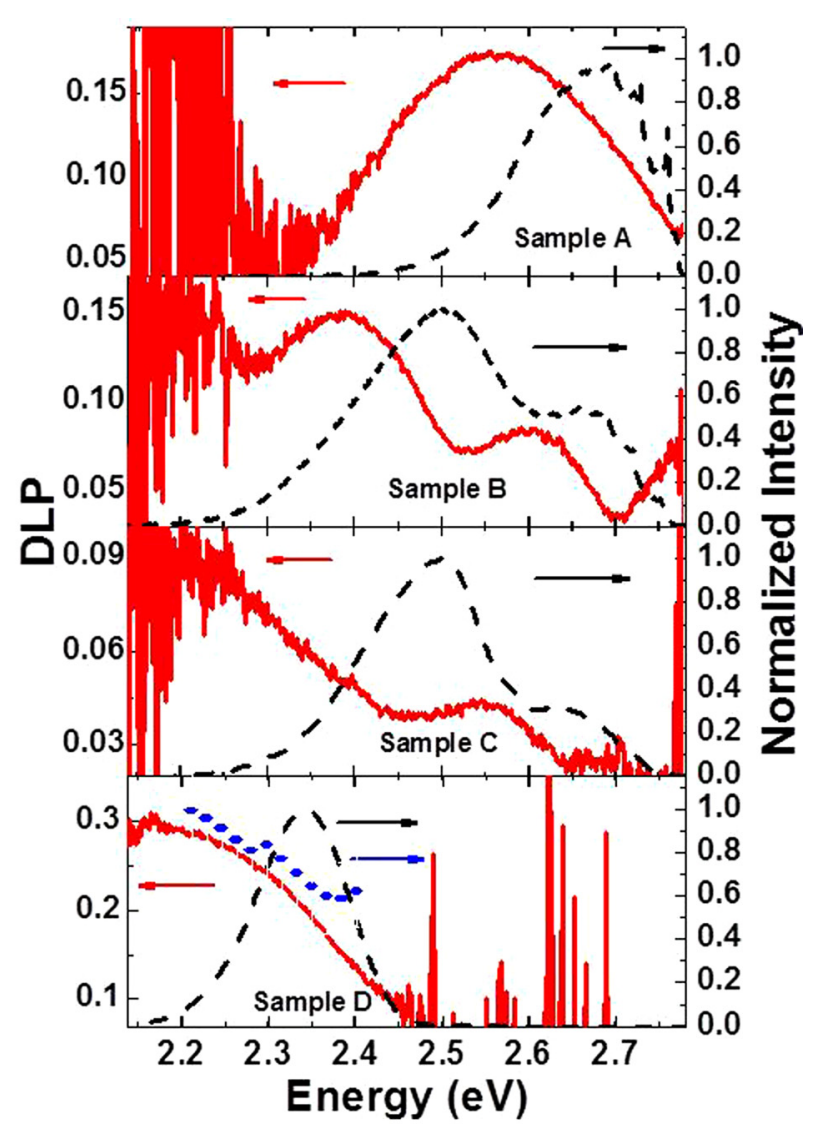

FIG. 3. Spectral dependence of the degree of linear polarization for all samples overlaid over corresponding normalized spectra (dashed lines). The blue dots are the spectral dependence of normalized anisotropic exchange splitting for sample D.

references therein) revealed that the PL of the QDs (generally seen as broad emission with energy $<2.6 \mathrm{eV}$ ) is convoluted with the emission (energy $>2.6 \mathrm{eV}$ ) from excitons bound to $\mathrm{Te}_{\mathrm{n} \geq 2}$ isoelectronic centers (ICs) within the $\mathrm{ZnSe}$ barriers. ${ }^{32}$ With the increase of Te flux, the PL spectrum changes gradually from an IC dominated emission (sample A) to a QD dominated emission (sample D), indicating the increase of the QD density. ${ }^{34,39}$ In addition, the red shift of energy of the QD emission bands, from samples B and C to sample D, indicates that the size of the QDs increases with increasing Te flux. ${ }^{33,34,40}$ There are several facts in the spectral dependence of a DLP suggesting that instead of anisotropic strain relief or defects it is the QDs structural elongation which dominates to the optical anisotropy.

First, for samples B-D, the QD related PL is more linearly polarized than the IC related emission. This trend agrees with the spectral dependence of anisotropic exchange splitting for sample D obtained (as discussed above) from the $D C P$ for different emission energies, shown as blue dots in Figure 3. Second, the overall DLP of sample A, whose PL is dominated by IC related emission, is smaller than that of sample D, whose PL is dominated by QD related emission. Indeed, sample D has the highest DLP among all the samples, correlated with the highest Te concentration and the highest QD density. ${ }^{34}$ In addition, samples $B$ and $C$ have similar Te content, PL spectrum, and spectral dependence of $D L P$. At the same time, sample $\mathrm{B}$ is characterized by the 

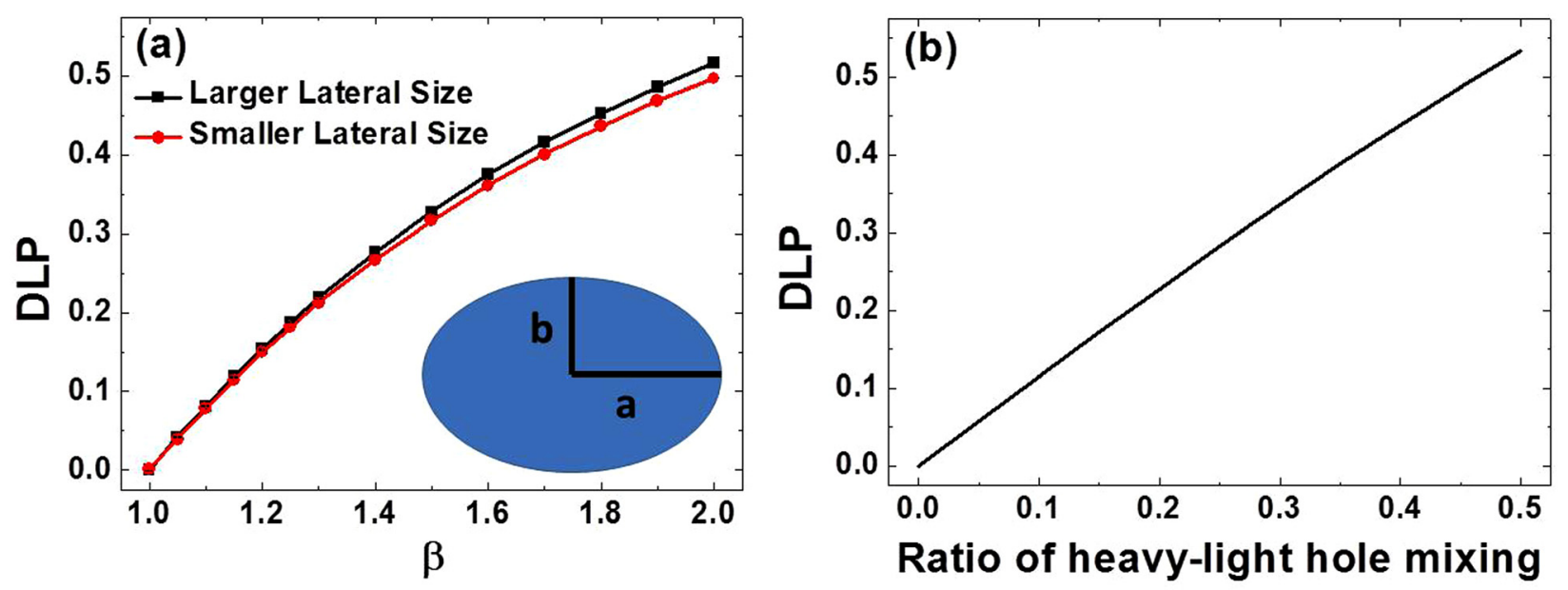

FIG. 4. (a) Calculated $D L P$ as a function of aspect ratio of the elongated QDs with larger lateral size (squares) and smaller lateral size (circles) as described in text. The inset shows a lateral elongated QD with aspect ratio $\beta=a / b$. (b) $D L P$ as a function of the ratio of heavy-light hole mixing calculated from Eq. (2).

lowest strain, whereas sample $\mathrm{C}$ has the highest strain among the four samples. It is also noticeable that both samples B and C have two "humps" at similar emission energies for spectral DLP, correlated with the stacked nature of ZnTe QDs. ${ }^{30,40}$ All the above features point to the elongation of the ZnTe QDs as a leading cause of the optical anisotropy rather than the anisotropic strain relief or defects in $\mathrm{ZnSe}$ barrier.

To estimate lateral shape anisotropy of the QDs, we applied the theory developed in Refs. 23 and 25, where the elongation of QDs that causes the linear polarization of the $\mathrm{PL}$ is explained by the difference in the occupation probabilities of holes in $p_{x}$ and $p_{y}$ orbitals, which is expressed as

$$
D L P=\frac{\left|\psi_{h}^{x}\right|^{2}-\left|\psi_{h}^{y}\right|^{2}}{\left|\psi_{h}^{x}\right|^{2}+\left|\psi_{h}^{y}\right|^{2}} .
$$

Here, $\psi_{h}^{x}=\left\langle x \mid \Psi_{h}\right\rangle$ and $\psi_{h}^{y}=\left\langle y \mid \Psi_{h}\right\rangle$ are the envelop functions, which represent the components of the wavefunction of hole $\Psi_{h}$ in $p_{x}$ orbit state $|x\rangle$ and $p_{y}$ orbit state $|y\rangle$, respectively. We used COMSOL to calculate the ground state wavefunction of holes confined in an elliptical ZnTe QD and the corresponding envelop functions. For this purpose, we interpolated band parameters, such as valence band offset, effective masses of hole and dielectric constants of ZnTe/ ZnSe QDs taken from Ref. 41. The calculations were done for QDs with areas of $a \times b=388$ and $231 \mathrm{~nm}^{2}$ (here $a$ and $b$ are the major and minor axes of the ellipse, respectively), which correspond to the average lateral size of samples D, B, and $C,{ }^{34,40}$ respectively. The results in terms of the aspect ratio $\beta=a / b$ are shown in Figure 4(a).

Comparing with the spectral results of DLP shown in Figure 3, we conclude that the aspect ratio of the QDs in sample D ranges from 1.2 to 1.4 , while the aspect ratio of the QDs in samples B and C ranges from 1.1 to 1.2 and from 1.05 to 1.1 , respectively. In the last section of Ref. 23 , the local strain profile due to possible structural anisotropy of QDs is calculated and comes out as positive contribution to the optical anisotropy. Therefore, the aspect ratios we calculated above must be treated as the upper limits in our real
QD systems. We nonetheless point out that the obtained aspect ratios are also in a good agreement with those obtained via HRXRD experiments. ${ }^{35}$ Quantum dots in sample D are somewhat thicker than those in samples B and C. ${ }^{34,39}$ This suggests that the thicker QDs have larger aspect ratios.

To further investigate this and the ratio of heavy-light hole mixing in different samples and its relation to the QD size, we plot the $D L P$ as a function of $\tilde{\gamma}$ calculated from Eq. (2) in Figure 4(b). The monotonic dependences of the DLP on both aspect ratio and heavy-light hole mixing ratio indicate that the heavy-light hole mixing is enhanced by the increase of structural anisotropy, agreeing with the discussions in Refs. 8-11. Comparing with the spectral results of $D L P$, we find out that the ratio of heavy-light mixing ranges from 0.04 to 0.26 in various samples, with larger (thicker) QDs having stronger heavy-light hole mixing. This conclusion agrees with the discussion in Ref. 8 that the ratio of heavy-light hole mixing is inversely proportional to the energy separation between the heavy hole and light hole ground states, based on the facts that smaller QDs have stronger confinement for holes which leads to larger energy separations between heavy and light holes.

In summary, we studied the optical anisotropy of type-II ZnTe/ZnSe submonolayer QDs. The ratio of heavy-light hole mixing is found to be $\sim 0.16$ from the $D L P$ of sample $\mathrm{D}$. The anisotropic exchange splitting extracted from the field dependence of $D C P$ gives a value of about $200 \mu \mathrm{eV}$. Through analysis of spectral dependence of DLP in four QD samples, we propose that the optical anisotropy is mostly related to structure elongation of the ZnTe QDs, instead of the anisotropic strain relief and defects in ZnSe barrier or any interfacial symmetry lowering. We calculated the aspect ratios of the QDs in samples B, C, and D, which show thicker QDs are more elongated while having stronger heavy-light hole mixing.

\section{ACKNOWLEDGMENTS}

The work was supported by the U.S. Department of Energy Office of Basic Energy Sciences, Division of Materials Sciences and Engineering under Award No. DESC003739. A portion of this work was performed at the 
National High Magnetic Field Laboratory, which was supported by National Science Foundation Cooperative Agreement No. DMR-1157490 and the State of Florida. J.L. and D.S. also acknowledge the support by NHMFL UCGP No. 5087. H.J. and I.L.K. also acknowledge NSF under Award No. DMR-1006050 for the work related to $D C P$.

${ }^{1}$ A. M. Fischer, V. L. Campo, Jr., M. E. Portnoi, and R. A. Römer, Phys. Rev. Lett. 102, 096405 (2009).

${ }^{2}$ F. Ding, N. Akopian, B. Li, U. Perinetti, A. Govorov, F. M. Peeters, C. C. Bof Bufon, C. Deneke, Y. H. Chen, A. Rastelli, O. G. Schmidt, and V. Zwiller, Phys. Rev. B 82, 075309 (2010).

${ }^{3}$ B. Li and F. M. Peeters, Phys. Rev. B 83, 115448 (2011).

${ }^{4}$ D. Loss and D. P. DiVincenzo, Phys. Rev. A 57, 120 (1998).

${ }^{5}$ A. Shabaev, Al. L. Efros, D. Gammon, and I. A. Merkulov, Phys. Rev. B 68, 201305(R) (2003).

${ }^{6}$ D. Bimberg, M. Grundmann, and N. N. Ledentsov, Quantum Dot Heterostructures (Wiley, New York, 1998).

${ }^{7}$ M. Usman, J. Appl. Phys. 110, 094512 (2011).

${ }^{8}$ A. V. Koudinov, I. A. Akimov, Yu. G. Kusrayev, and F. Henneberger, Phys. Rev. B 70, 241305(R) (2004).

${ }^{9}$ D. N. Krizhanovskii, A. Ebbens, A. I. Tartakovskii, F. Pulizzi, T. Wright, M. S. Skolnick, and M. Hopkinson, Phys. Rev. B 72, 161312(R), (2005).

${ }^{10}$ C. Tonin, R. Hostein, V. Voliotis, R. Grousson, A. Lemaitre, and A. Martinez, Phys. Rev. B 85, 155303 (2012).

${ }^{11}$ Yu. I. Mazur, S. Noda, G. G. Tarasov, V. G. Dorogan, G. J. Salamo, O. Bierwagen, W. T. Masselink, E. A. Decuir, Jr., and M. O. Manasreh, J. Appl. Phys. 103, 054315 (2008).

${ }^{12}$ R. I. Dzhioev, H. M. Gibbs, E. L. Ivchenko, G. Khitrova, V. L. Korenev, M. N. Tkachuk, and B. P. Zakharchenya, Phys. Rev. B 56, 13405 (1997).

${ }^{13}$ R. Seguin, A. Schliwa, S. Rodt, K. Potschke, U. W. Pohl, and D. Bimberg, Phys. Rev. Lett. 95, 257402 (2005).

${ }^{14}$ C. Lin, W. You, H. Chou, S. Cheng, S. Lin, and W. Chang, Phys. Rev. B 83, 075317 (2011).

${ }^{15}$ M. Molas, K. Gołasa, K. Kuldová, J. Borysiuk, A. Babiński, J. Lapointe, and Z. R. Wasilewski, J. Appl. Phys. 111, 033510 (2012).

${ }^{16}$ Zh. M. Wang, H. Churchill, C. E. George, and G. J. Salamo, J. Appl. Phys. 96, 6908 (2004).

${ }^{17}$ C. Hermannstädter, M. Witzany, M. Heldmaier, R. Hafenbrak, K. D. Jöns, G. J. Beirne, and P. Michler, J. Appl. Phys. 111, 063526 (2012).

${ }^{18}$ M. Sugisaki, H. Ren, S. V. Nair, K. Nishi, S. Sugou, T. Okuno, and Y. Masumoto, Phys. Rev. B 59, R5300 (1999).
${ }^{19}$ M. Schmidbauer, F. Hatami, M. Hanke, P. Schafer, K. Braune, W. T. Masselink, R. Kohler, and M. Ramsteiner, Phys. Rev. B 65, 125320 (2002).

${ }^{20}$ L. Besombes, L. Marsal, K. Kheng, T. Charvolin, Le Si Dang, A. Wasiela, and H. Mariette, J. Cryst. Growth 214/215, 742-746 (2000).

${ }^{21}$ T. Kiessling, G. V. Astakhov, A. V. Platonov, T. Slobodskyy, S. Mahapatra, W. Ossau, G. Schmidt, K. Brunner, and L. W. Molenkamp, Phys. Status Solidi C 3(4), 912-915 (2006).

${ }^{22}$ Z. Xu, D. Birkedal, J. M. Hvam, Z. Zhao, Y. Liu, K. Yang, A. Kanjilal, and J. Sadowski, Appl. Phys. Lett. 82, 3859 (2003).

${ }^{23}$ W. Sheng, Appl. Phys. Lett. 89, 173129 (2006).

${ }^{24}$ M. Usman, Phys. Rev. B 86, 155444 (2012).

${ }^{25}$ W. Sheng and S. J. Xu, Phys. Rev. B 77, 113305 (2008).

${ }^{26}$ L. Fortunato, M. T. Todaro, V. Tasco, M. De Giorgi, M. De Vittorio, R. Cingolani, and A. Passaseo, Superlattices Microstruct. 47, 72 (2010).

${ }^{27}$ D. Alonso-Álvarez, J. María Ripalda, B. Alén, J. M. Llorens, A. Rivera, and F. Briones, Adv. Mater. 23, 5256 (2011).

${ }^{28}$ M. Grundmann, O. Stier, and D. Bimberg, Phys. Rev. B 52, 11969 (1995).

${ }^{29}$ E. L. Ivchenko and M. O. Nestoklon, Phys. Rev. B 70, 235332 (2004).

${ }^{30}$ Y. Gong, W. MacDonald, G. F. Neumark, M. C. Tamargo, and I. L. Kuskovsky, Phys. Rev. B 77, 155314 (2008).

${ }^{31}$ Y. Gu, I. L. Kuskovsky, M. van der Voort, G. F. Neumark, X. Zhou, and M. C. Tamargo, Phys. Rev. B 71, 045340 (2005).

${ }^{32}$ J. Phillips, J. Appl. Phys. 91, 4590 (2002).

${ }^{33}$ H. Ji, B. Roy, S. Dhomkar, R. T. Moug, M. C. Tamargo, A. Wang, and I. L. Kuskovsky, J. Electron. Mater. 42, 3297 (2013).

${ }^{34}$ S. Dhomkar, H. Ji, B. Roy, V. Deligiannakis, A. Wang, M. C. Tamargo, and I. L. Kuskovsky, J. Cryst. Growth 422, 8-14 (2015).

${ }^{35}$ S. Dhomkar, N. Vaxelaire, H. Ji, V. Shuvayev, M. C. Tamargo, I. L. Kuskovsky, and I. C. Noyan, Appl. Phys. Lett. 107, 251905 (2015).

${ }^{36}$ B. Roy, A. D. Shen, M. C. Tamargo, and I. L. Kuskovsky, J. Electron. Mater. 40, 1775 (2011).

${ }^{37}$ S. Sancho, M. Chaouache, M. A. Maaref, F. Bernardot, B. Eble, A. Lemaître, and C. Testelin, Phys. Rev. B 84, 155458 (2011).

${ }^{38}$ H. Ji, S. Dhomkar, R. Wu, J. Ludwig, Z. Lu, D. Smirnov, M. C. Tamargo, G. W. Bryant, and I. L. Kuskovsky, "Long Spin-Flip Time and Large Zeeman Splitting of Holes in Type-II ZnTe/ZnSe Submonolayer Quantum Dots," Phys. Rev. B (to be published).

${ }^{39}$ I. L. Kuskovsky, Y. Gu, M. van der Voort, G. F. Neumark, X. Zhou, M. Munoz, and M. C. Tamargo, Phys. Status Solidi 241, 527 (2004).

${ }^{40}$ H. Ji, S. Dhomkar, B. Roy, V. Shuvayev, V. Deligiannakis, M. C. Tamargo, J. Ludwig, D. Smirnov, A. Wang, and I. L. Kuskovsky, J. Appl. Phys. 116, 164308 (2014).

${ }^{41}$ V. A. Shuvayev, I. L. Kuskovsky, L. I. Deych, Y. Gu, Y. Gong, G. F. Neumark, M. C. Tamargo, and A. A. Lisyansky, Phys. Rev. B 79, 115307 (2009). 